\title{
Eficiência da correção endoventricular com patch em pacientes com grande área acinética pós-infarto do miocárdio e severa disfunção ventricular
}

\author{
Gustavo Calado de Aguiar RIBEIRO*, Cledicyon Eloy COSTA*, Maurício Marson LOPES*, \\ Ana Paula Nunes de ALBUQUERQUE* ${ }^{*}$ José Pedro da SILVA*
}

RBCCV 44205-567

\begin{abstract}
Ribeiro G C A, Costa C E, Lopes M M, Albuquerque A P N, Silva J P - Eficiência da correção endoventricular com patch em pacientes com grande área acinética pós-infarto do miocárdio e severa disfunção ventricular. Rev Bras Cir Cardiovasc 2002; 17(1): 13-8.
\end{abstract}

RESUMO: Objetivo: Verificar a eficiência da técnica de plastia endoventricular com patch circular nos casos com grande área acinética causada por infarto agudo do miocárdio prévio no ventrículo esquerdo e com severa depressão da função ventricular.

Casuística e Métodos: Este estudo evoluiu resultados hemodinâmicos da plastia endoventricular com patch circular em pacientes com grande área acinética ( 9 casos) e grande área discinética (11 casos) e ambos apresentando severa depressão ventricular (fração de ejeção < 35\%). A distinção destes dois grupos foi feita através de ventriculografia radioisotópica. Os grupos foram comparados por ecocardiografia e estudo hemodinâmico através de cateter de Swan-Ganz no pré e pós-operatório.

Resultados: Os resultados mostraram uma imediata melhora na classe funcional, melhora estatisticamente significativa (teste $T$ de Student) na fração de ejeção (de $25 \pm 0,8 \%$ para $39 \pm 1,6 \%$ nos acinéticos e de $27 \pm 0,7 \%$ para $41 \pm 1,6 \%$ nos discinéticos), diminuição do capilar pulmonar $(20 \pm 1,2 \mathrm{mmHg}$ para $12 \pm 1,2 \mathrm{mmHg}$ nos acinéticos e de $17 \pm 0,5 \mathrm{mmHg}$ para $11 \pm 0,9 \mathrm{mmHg}$ nos discinéticos), diminuição dos índices volumes sistólico (de $176 \pm 9,2 \mathrm{ml}$ para $77 \pm 6,2 \mathrm{ml}$ no acinéticos e de $160 \pm 10 \mathrm{ml}$ para $66 \pm 2 \mathrm{ml}$ nos discinéticos) e diastólico (de $226 \pm 11 \mathrm{ml}$ para $115 \pm 8 \mathrm{ml}$ no acinéticos e de $209 \pm$ $11 \mathrm{ml}$ para $96 \pm 5,0 \mathrm{ml}$ nos discinéticos) final do ventrículo esquerdo. A pressão arterial pulmonar também diminuiu, embora não significativa. A mortalidade global foi de $10 \%$, sendo 2 óbitos no grupo acinesia $(22,2 \%)$ e nenhum no outro grupo.

Conclusão: Os resultados imediatos obtidos são comparáveis aos descritos na literatura. Acreditamos que a técnica de reconstrução endoventricular com patch circular seja efetiva e possa ser usada como opção cirúrgica para casos de extensa disfunção cardíaca e grande área acinética.

DESCRITORES: Infarto do miocárdio, complicações. Aneurismas cardíacos, cirurgia. Ventrículo cardíaco, cirurgia. Função ventricular.

\footnotetext{
Trabalho realizado no Serviço de Cirurgia Cardíaca do Hospital Samaritano de Campinas e Hospital Irmãos Penteado de Campinas. SP, Brasil. *Do Hospital Samaritano e do Hospital Irmãos Penteado de Campinas.

Recebido para publicação em julho de 2001.

Endereço para correspondência: Rua Júlio de Mesquita, 571. Cambuí. Campinas, SP, Brasil. CEP: 13015-130. Tel. (19) 3232-3856.

e.mail: cardiocirurgica@uol.com.br
} 
Ribeiro G C A, Costa C E, Lopes M M, Albuquerque A P N, Silva J P - Eficiência da correção endoventricular com patch em pacientes com grande área acinética pós-infarto do miocárdio e severa disfunção ventricular. Rev Bras Cir Cardiovasc 2002; 17(1): 13-8.

\section{INTRODUÇÃO}

O aneurisma de ventrículo esquerdo (Aneu VE) é a complicação mecânica mais freqüente após o infarto agudo do miocárdio e sua ressecção cirúrgica continua sendo o tratamento de escolha para aqueles que apresentam sintomas (angina, insuficiência cardíaca congestiva, arritmias e tromboembolismo). Esta aceitação ganhou maior ênfase a partir de meados da década de 80, quando JATENE (1) introduziu o conceito de correção geométrica destes aneurismas, sendo seguido por DOR et al. ${ }^{(2)} \mathrm{e}$ COOLEY (3).

Essa região cicatricial do ventrículo esquerdo (Aneu VE) pode apresentar uma movimentação sistólica paradoxal (discinesia) ou mesmo não ter movimento (acinesia). Tem sido destacada na literatura uma evolução menos favorável para pacientes que apresentam acinesia, principalmente quando essa área acinética for grande e a função ventricular global se encontrar deteriorada.

Neste estudo, procuramos avaliar a eficácia da técnica descrita por DOR et al. (4) a plastia com patch endoventricular, em pacientes com grande área acinética e com severa depressão da função ventricular, comparando com pacientes que foram submetidos a correção de áreas discinéticas e também deteriorada função cardíaca.

\section{CASUÍSTICA E MÉTODOS}

No período de janeiro de 1997 a janeiro de 2000, 41 pacientes foram submetidos à correção cirúrgica de Aneu VE pelo nosso Serviço.

$\mathrm{Na}$ seleção de casos, 21 pacientes foram retirados do estudo por não terem sido operados pela técnica de correção descrita por DOR et al. (4), por apresentarem fração de ejeção pré-operatória maior que $35 \%$ ou por não apresentarem dados completos.

Os pacientes incluídos (20 casos) sofreram infarto agudo do miocárdio anterior e tinham Aneu VE ântero-apical e fração de ejeção menor que $35 \%$.

Submeteram-se a cinecoronariografia, ventriculografia, ecocardiografia, avaliação de viabilidade miocárdica por ventriculografia radioisotópica, e estudo hemodinâmico com cateter de Swan-Ganz. No pós-operatório, foram repetidas as medidas hemodinâmicas através do cateter de Swan-Ganz e ecocardiografia. A distinção entre acinesia e discinesia foi feita pela ventriculografia radioisotópica por análise qualitativa e imagem paramétrica de fase e posteriormente, os pacientes foram separados em dois grupos. No grupo A, 9 pacientes com acinesia e, no grupo B, 11 pacientes com discinesia. Os dados pré-operatórios estão na Tabela 1.

Todos os pacientes foram operados com sistema de circulação extracorpórea a $34^{\circ} \mathrm{C}$. Foi usada cardioplegia sangüínea hipotérmica, enriquecida por

TABELA 1

\begin{tabular}{|c|c|c|c|}
\hline \multicolumn{4}{|c|}{ DADOS PRÉ-OPERATÓRIOS } \\
\hline & $\begin{array}{c}\text { ACINESIA } \\
\text { (9 pacientes) }\end{array}$ & $\begin{array}{c}\text { DISCINESIA } \\
\text { (11 pacientes) }\end{array}$ & $\begin{array}{c}\text { TOTAL } \\
20\end{array}$ \\
\hline Idade (anos) & $63 \pm 1,3$ & $59 \pm 1,4$ & \\
\hline Sexo (masculino) & $6 / 3$ & $7 / 4$ & \\
\hline Dt do IAM (meses) & $32 / 3 m$ & $27 / 2 m$ & \\
\hline \multicolumn{4}{|l|}{ Angina } \\
\hline Sim & $4(44,4 \%)$ & $7(63,6 \%)$ & \\
\hline Não & $5(55,6 \%)$ & $4(36,6 \%)$ & \\
\hline \multicolumn{4}{|l|}{ NYHA classe } \\
\hline$|-| \mid$ & $3(33,3 \%)$ & $4(36,6 \%)$ & \\
\hline III-IV & $6(66,6 \%)$ & $7(63,6 \%)$ & \\
\hline Casos em urgência / emergência & $1(11,1 \%)$ & $2(18,1 \%)$ & \\
\hline \multicolumn{4}{|l|}{ Extensão da DAC } \\
\hline Uniarterial & $1(11,1 \%)$ & $2(18,1 \%)$ & \\
\hline 2 vasos & $5(55,5 \%)$ & $4(36,3 \%)$ & \\
\hline 3 vasos & $3(33,3 \%)$ & $5(45,4 \%)$ & \\
\hline TCE & 0 & $1(9,09 \%)$ & \\
\hline
\end{tabular}

IAM - infarto agudo do miocárdio; NYHA - New York Heart Association; DAC - doença arterial coronariana; Dt IAM - tempo em meses decorrente após IAM; TCE - lesão em tronco de artéria coronária esquerda. 
Ribeiro G C A, Costa C E, Lopes M M, Albuquerque A P N, Silva J P - Eficiência da correção endoventricular com patch em pacientes com grande área acinética pós-infarto do miocárdio e severa disfunção ventricular. Rev Bras Cir Cardiovasc 2002; 17(1): 13-8.

aspartato e glutamato, infundida por via anterógrada e retrógrada. A técnica cirúrgica usada, plastia endoventricular com patch de Dacron (Hemashield: Meadox Medical, Inc, Oakland), foi descrita por DOR et al. (4), em 1984.

A revascularização do miocárdio foi empregada sempre que indicada, usando-se como enxertos a artéria torácica interna e veia safena.

\section{RESULTADOS}

Os dados referentes ao procedimento cirúrgico encontram-se na Tabela 2.

Tivemos um maior tempo de perfusão e de pinçamento aórtico no grupo A. Somente em 1 paciente não foi feita revascularização do miocárdio, sendo este do grupo $\mathrm{B}$. O uso de artéria mamária e o número de anastomoses distais foram similares nos dois grupos. A mortalidade hospitalar foi de $10 \%$, sendo 2 óbitos no grupo de acinesia (2/9 pacientes) e nenhum no outro grupo.

Os dados hemodinâmicos colhidos por ecocardiografia e monitorização por cateter de Swan-Ganz no pré e pós-operatório estão na Tabela 3 .
Os dados estatísticos (teste $\mathrm{T}$ de Student) demonstraram melhora na medida do capilar pulmonar, na fração de ejeção, índice de volume diastólico e sistólico finais quando foram comparados com os dados basais de cada grupo.

\section{COMENTÁRIOS}

O seguimento clínico de pacientes com Aneu VE mostra maior sobrevida para os pacientes que se comportam clinicamente assintomáticos. GRONDIN et al. ${ }^{(5)}$, em 1979, mostravam uma sobrevida de $90 \%$ em 5 anos para pacientes com Aneu VE em assintomáticos, e apenas $47 \%$ de sobrevida para os sintomáticos no mesmo intervalo de tempo. Entretanto, o estudo CASS (6) apontou um prognóstico significativamente pior para indivíduos com Aneu VE com pobre função ventricular e extensa doença coronariana, a despeito da sintomatologia.

Assim, com estes dados, houve uma ampliação na indicação cirúrgica de pacientes portadores de Aneu VE, impulsionada, principalmente, pela introdução de novas técnicas de reconstrução do ventrículo de forma geométrica por JATENE (1), DOR et al. (2) e COOLEY (3).

TABELA 2

\begin{tabular}{lcc}
\hline & DADOS DO PROCEDIMENTO CIRÚRGICO & \\
\hline & $\begin{array}{c}\text { ACINÉTICOS } \\
\text { (9 pacientes) }\end{array}$ & $\begin{array}{c}\text { DISCINÉTICOS } \\
\text { (11 pacientes) }\end{array}$ \\
Tempo de perfusão (min) & $119,2 \pm 15,1$ & $100,9 \pm 6,8$ \\
Tempo de pinçamento aórtico (min) & 83 & 56 \\
Procedimento em mitral & 0 & $1(11,1 \%)$ \\
Uso de artéria mamária & $88,80 \%$ & $90,90 \%$ \\
Números de enxertos distais & 2,20 & 2,18 \\
Mortalidade hospitalar & $2(22,2 \%)$ & 0 \\
Uso de BIA & $6(66,6 \%)$ & $6(54,4 \%)$ \\
\hline
\end{tabular}

BIA: balão intra-aórtico.

TABELA 3

\begin{tabular}{lcccc}
\hline & \multicolumn{2}{c}{$\begin{array}{c}\text { DADOS HEMODINÂMICOS COLHIDOS POR ECOCARDIOGRAFIA E PELO } \\
\text { CATETER DE SWAN-GANZ }\end{array}$} \\
\hline & \multicolumn{2}{c}{$\begin{array}{c}\text { ACINÉTICOS } \\
\text { (9 pacientes) }\end{array}$} & \multicolumn{2}{c}{$\begin{array}{c}\text { DISCINÉTICOS } \\
\text { (11 pacientes) }\end{array}$} \\
& pré & pós-operatório & pré & pós-operatório \\
Capilar pulmonar & $20 \pm 1,2$ & $12 \pm 1,2^{*}$ & $17 \pm 0,5$ & $11 \pm 0,9^{*}$ \\
Pressão arterial pulmonar & $26 \pm 1,3$ & $21 \pm 1,4$ & $24 \pm 1,5$ & $18 \pm 1,3$ \\
Fração de ejeção & $25 \pm 0,8$ & $39 \pm 1,6^{*}$ & $27 \pm 0,7$ & $41 \pm 1,6^{*}$ \\
VDFVEI ml/m & $226 \pm 11$ & $115 \pm 7,8^{*}$ & $209 \pm 11$ & $96 \pm 5,0^{*}$ \\
VSFVEI ml/m & $176 \pm 9,2$ & $77 \pm 6,2^{*}$ & $160 \pm 10$ & $66 \pm 2^{*}$ \\
\hline
\end{tabular}

VDFVEI - índice de volume diastólico final do ventrículo esquerdo; VSFVEI - índice de volume sistólico do ventrículo esquerdo.

*Mostra onde houve diferença estatística $(p<0,05)$ quando foram comparados os valores pré e pós-operatórios. 
Ribeiro G C A, Costa C E, Lopes M M, Albuquerque A P N, Silva J P - Eficiência da correção endoventricular com patch em pacientes com grande área acinética pós-infarto do miocárdio e severa disfunção ventricular. Rev Bras Cir Cardiovasc 2002; 17(1): 13-8.

Os resultados apresentados por JATENE (7), em 1381 pacientes com mortalidade imediata de apenas $5,8 \%$ e tardia de $4.5 \%$ e por DOR et al. ${ }^{(8)} \mathrm{em}$ 715 pacientes com mortalidade em 30 dias de $7 \%$, sendo que $34 \%$ destes pacientes eram portadores de grave disfunção ventricular, fortaleceram a extensão da indicação cirúrgica.

Resultados concordantes de vários autores apontam uma evolução mais favorável naqueles que apresentavam discinesia e foram submetidos a aneurismectomia. MANGSCHAU (9) encontrou meIhora da classe funcional (New York Heart Association) e uma maior tolerância ao exercício quando comparou discinesia e acinesia, estas diagnosticadas por ventriculografia radioisotópica.

COUPER et al. (10), analisando 303 portadores de Aneu VE num seguimento de 5 anos, encontraram menor mortalidade operatória ( $8 \%$ versus $18 \%$ ) para pacientes com discinesia e maior sobrevida $63 \%$ contra $51 \%$ em acinéticos.

DI DONATO et al. (11), em uma série de 245 pacientes (125 com discinesia e $120 \mathrm{com}$ acinesia), usando a técnica descrita por Dor, dividiram-nos em dois grupos, de acordo com a extensão da área comprometida do ventrículo esquerdo (área comprometida maior ou menor que $60 \%$ ). Encontraram uma mortalidade de $12,5 \%$ para casos de acinesia com grande área comprometida e nula para aqueles de acinesia com pequena área comprometida.

$O$ determinante na diferente evolução entre pacientes com acinesia e com discinesia não é atividade mecânica dessa área infartada, mais sim, a atividade mecânica da parte não infartada imediatamente adjacente à área lesionada. A função sistólica desses segmentos adjacentes está preservada e rapidamente diferencia-se do segmento infartado nos casos de pacientes com discinesia. Ao contrário, pacientes com acinesia apresentam esses segmentos afinados, remodelados e hipocontrácteis que se misturam com a área lesada. Esses casos com acinesia tipicamente têm função ventricular ruim (Figura 1) e clinicamente têm insuficiência cardíaca congestiva.

Recentemente, face ao aumento da população coronariana em estágio final de doença isquêmica e limitado número de doadores para transplante cardíaco, terapias alternativas para alívio dos sintomas e aumento da sobrevida têm sido exploradas. Neste contexto, DOR ${ }^{(12)}$ e MICKEBOROUG ${ }^{(13)}$ têm proposto excisão do aneurisma que apresenta comprometimento global da função ventricular e também com difícil distinção do segmento miocárdio ao aneurisma e este.

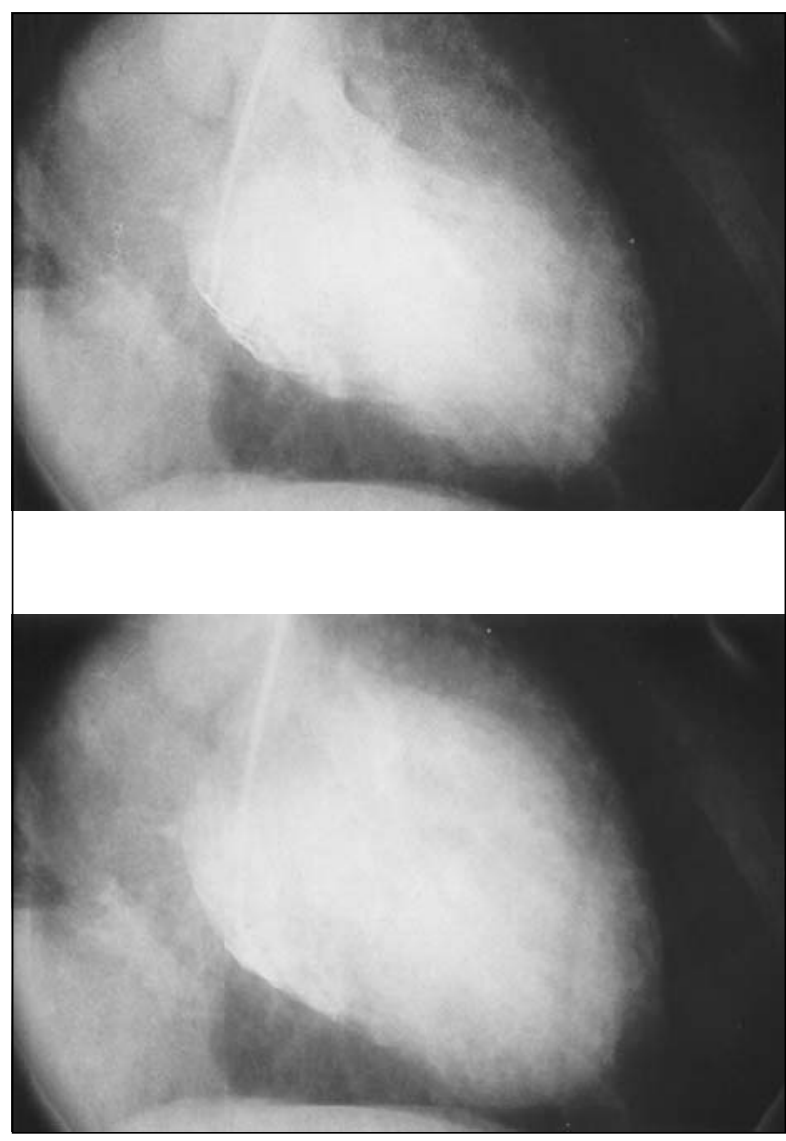

Fig. 1 - Fotos da ventriculografia do coração em sístole e diástole.

Deparamos-nos com pacientes mostrando grave disfunção ventricular e acinesia e, frente à restrição do número de doadores para transplante cardíaco, procuramos uma alternativa cirúrgica. A redução de volume, através de cardiectomia lateral proposta por Batista, não se aplica para esses pacientes onde a área lesada é anterior e septal, e a área lateral não é isquêmica e fibrosada. A cardiomioplastia somente previne dilatação futura, não reduzindo o tamanho da câmara, além do que não apresenta resultados imediatos e, ainda, muitos dos pacientes têm critérios de contra-indicação, como a classe funcional (NYHA IV).

DOR et al. (14) apontam como vantagens do patch endoventricular para esses casos: (a) reconstrução geométrica; (b) permitir a exclusão de áreas não fibróticas não ressecadas, como o septo ventricular; (c) redução da cavidade ventricular; (d) revascularização de todos os territórios, o que melhora a contratilidade e diminui a ocorrência de arritmias. 
Ribeiro G C A, Costa C E, Lopes M M, Albuquerque A P N, Silva J P - Eficiência da correção endoventricular com patch em pacientes com grande área acinética pós-infarto do miocárdio e severa disfunção ventricular. Rev Bras Cir Cardiovasc 2002; 17(1): 13-8.

Nos nossos pacientes, encontramos resultados próximos aos da literatura quanto à mortalidade hospitalar $(22,2 \%$ no grupo com acinesia e $10 \%$ no geral), apesar do número restrito de pacientes. Esses óbitos ocorreram por falência de múltiplos órgãos decorrente de choque cardiogênico e o outro por complicações pulmonares. Quanto aos dados hemodinâmicos, percebemos uma melhora expressiva nos dois grupos com diminuição dos volumes sistólico (Figura 2) e diastólico final do ventrículo esquerdo.

O uso de balão intra-aórtico foi maior do que o encontrado na literatura, justificado pelo quadro grave destes pacientes com rebaixada fração de ejeção e alta pressão capilar pulmonar.

Houve melhora clínica significativa para os dois grupos de pacientes e melhora da fração de ejeção (23\% para $39 \%$ ) demonstrada ao ecocardiograma de controle na alta hospitalar.

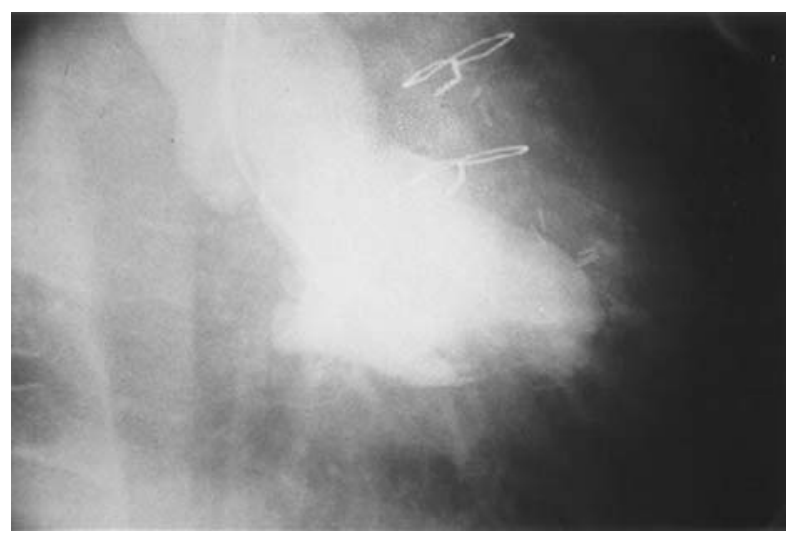

Fig. 2-Foto de ventriculografia em sístole em após correção cirúrgica.

Concluímos que a técnica de reconstrução ventricular com patch endoventricular, descrita por Dor, para aneurisma é eficiente e pode ser uma alternativa para pacientes com evolução clínica desfavorável.

Ribeiro G C A, Costa C E, Lopes M M, Albuquerque A P N, Silva J P - Efficacy of endoventricular patch plasty in large postinfarction akinetic scar and severe left ventricular dysfunction. Rev Bras Cir Cardiovasc 2002; 17(1): 13-8.

ABSTRACT: Purpose: Efficacy of endoventricular patch plasty in large postinfarction akinetic scar and severe left ventricular dysfunction.

Methods: This study evaluated clinical and hemodynamic results of endoventricular circular patch plasty in patients with either large akinetic scar $(n=9)$ or large dyskinetic scar $(n=11)$ and depressed left ventricular function (ejection fraction < 35\%). The difference between akinetic and dyskinetic left ventricular aneurysms was diagnosed by gated radionuclide ventriculography. Groups were comparable for symptons and by echocardiography and by hemodynamic before and after the surgery.

Results: Results showed an early improvement in New York Heart Association functional class. Statistically (the Student $t$ test) an improvement occurred in left ventricle ejection fraction (from $25 \pm$ $0.8 \%$ to $39 \pm 1.6 \%$ in akinetic patients and from $27 \pm 0.7 \%$ to $41 \pm 1.6 \%$ in dyskinetic patients), decreased of capillary wedge pressure $(20 \pm 1.2 \mathrm{mmHg}$ to $12 \pm 1.2 \mathrm{mmHg}$ in akinetics and $17 \pm 0,5 \mathrm{mmHg}$ to $11 \pm 0.9 \mathrm{mmHg}$ in dyskinetics patients), decrease end-diastolic volume index (226 $\pm 11 \mathrm{ml}$ to $115 \pm$ $7,8 \mathrm{ml}$ in akinetics and $209 \pm 11 \mathrm{ml}$ to $96 \pm 5 \mathrm{ml}$ in dyskinetics) and end-systolic volume index (176 $\pm 9.2 \mathrm{ml}$ to $77 \pm 6.2 \mathrm{ml}$ in akinetics and $160 \pm 10 \mathrm{ml}$ to $66 \pm 2 \mathrm{ml}$ in dyskinetics patients. Overall operative mortality was $10 \%$ ( 2 cases in akinetic group $=22.2 \%$ ).

Conclusion: We find that endoventricular circular patch plasty technique allows an effective correction and provides a significant improvement in cardiac function in patients with large postinfarction akinetic scar and severe left ventricular dysfunction.

DESCRIPTORS: Myocardial infarction, complications. Heart aneurysms, surgery. Heart ventricle, surgery. Ventricular function. 
Ribeiro G C A, Costa C E, Lopes M M, Albuquerque A P N, Silva J P - Eficiência da correção endoventricular com patch em pacientes com grande área acinética pós-infarto do miocárdio e severa disfunção ventricular. Rev Bras Cir Cardiovasc 2002; 17(1): 13-8.

\section{REFERÊNCIAS BIBLIOGRÁFICAS}

1 Jatene A D - Left ventricular aneurysmectomy: resection or reconstruction. J Thorac Cardiovasc Surg 1985; 89: $321-31$.

2 Dor V, Kreitmann P, Jourdan J et al. - Interest of physiological closure (circumferencial plasty on contractile areas) of left ventricle after resection and endocardectomy for aneurysm or akinetic zone: comparison with classic technique about a series of 209 left ventricular resections. J Cardiovasc Surg 1985; $26: 73$.

3 Cooley D A - Ventricular endoaneurysmorrhaphy: a simplified repair for extensive postinfarction aneurysm. J Card Surg 1989; 4: 200-5.

4 Dor V, Saad M, Coste P et al. - Left ventricular aneurysm: a new surgical approach. Thorac Cardiovasc Surg 1989; 37: 11-9.

5 Grondin P, Kretz J G, Bical O, Donzeau - George P, Petitclerc R, Campeau L - Natural history of saccular aneurysm of the left ventricle. $J$ Thorac Cardiovasc Surg 1979; 77: 57-64.

6 Faxon D P, Ryan T J, David K B et al. - Prognostic significance of angiographically documented left ventricular aneurysm from the coronary artery study (CASS). Am J Cardiol 1982; 50: 157-64.

7 Jatene A D - Surgical treatment of left ventricular aneurysm. In: Baue A E, Geha A S, Hammond G L, Laks H, Naunheim K S, eds. Glenn's thoracic and cardiovascular surgery. 5 ed, vol 2. Normalk: Appleton \& Lange Publishers, 1991: 1829-36.
8 Dor V, Sabatier M, Di Donato M et al. - Late hemodynamic results after left ventricular patch repair associated with coronary grafting in patients with post-infarction akinetic or dyskinetic aneurysm of the left ventricle. J Thorac Cardiovasc Surg 1995; 110: 1291-301.

$9 \quad$ Mangschau A - Akinetic versus dyskinetic left ventricular aneurysm diagnosed by gated scintigraphy: difference in surgical outcome. Ann Thorac Surg 1989; 47: 74651.

10 Couper G S, Bunton R W, Birjiniuk V et al. - Relative risks of left ventricular aneurysmectomy in patients with akinetic scars versus true dyskinetic aneurysms. Circulation 1990; 82 (5 suppl): IV 248-56.

11 Di Donato M, Sabatier M, Dor V, Toso A, Maioli M, Fantini $\mathrm{F}$ - Akinetic versus dyskinetic postinfarction scar: relation to surgical outcome in patients undergoing endoventricular circular patch plasty repair. J Am Coll Cardiol 1997; 29: 1569-75.

12 Dor V - Reconstructive left ventricular surgery for postischemic akinetic dilatation. Semin Thorac Cardiovasc Surg 1997; 9: 139-45.

13 Mickleborough $\mathrm{L} L$ - Left ventricular aneurysmectomy. In: Franco K L, Verrier E D, eds. Advanced therapy in cardiac surgery. Hamilton: BC Decker Inc, 1999. 347-53.

14 Dor V, Sabatier M, Di Donato M, Montiglio F, Tosa A, Maioli M - Efficacy of endoventricular patch plasty in large postinfarction akinetic scar and severe left ventricular dysfunction: comparison with a series of large dyskinetic scars. J Thorac Cardiovasc Surg; 116: $50-9$. 